\title{
Using the iterated sinh transformation to evaluate two dimensional nearly singular boundary element integrals
}

\author{
Peter R. Johnston *and Barbara M. Johnston, \\ School of Biomolecular and Physical Sciences, \\ Griffith University, \\ Nathan, \\ Queensland, \\ Australia, 4111, \\ Phone: 61-7-3735-7748, \\ Fax: 61-7-3735-7656, \\ E-mail: P.Johnston@griffith.edu.au \\ David Elliott, \\ School of Mathematics and Physics, \\ University of Tasmania, \\ Private Bag 37, \\ Hobart, \\ Tasmania, \\ Australia, 7001 \\ January 18, 2013
}

*Author to whom correspondence should be addressed. 


\begin{abstract}
Recently, sinh transformations have been proposed to evaluate nearly weakly singular integrals which arise in the boundary element method. These transformations have been applied to the evaluation of nearly weakly singular integrals arising in the solution of Laplace's equation in both two and three dimensions and have been shown to evaluate the integrals more accurately than existing techniques.

More recently, the sinh transformation was extended in an iterative fashion and shown to evaluate one dimensional nearly strongly singular integrals with a high degree of accuracy. Here the iterated sinh technique is extended to evaluate the two dimensional nearly singular integrals which arise as derivatives of the three dimensional boundary element kernel. The test integrals are evaluated for various basis functions and over flat elements as well as over curved elements forming part of a sphere.

It is found that two iterations of the sinh transformation can give relative errors which are one or two orders of magnitude smaller than existing methods when evaluating two dimensional nearly strongly singular integrals, especially with the source point very close to the element of integration. For two dimensional nearly weakly singular
\end{abstract}


integrals it is found that one iteration of the sinh transformation is sufficient.

Keywords: non-linear co-ordinate transformation; boundary element method; nearly singular integrals; numerical integration; sinh transformation. 


\section{Introduction}

The accurate evaluation of nearly singular two dimensional integrals is fundamental to a good implementation of the three dimensional boundary element method. Such integrals are "nearly" singular in the sense that the source point is close to, but not on, the element under consideration. A recently proposed sinh transformation has been shown to evaluate accurately nearly weakly singular integrals arising in both the two and three dimensional solutions of Laplace's equation using the boundary element method [14, 13]. More recently, the sinh transformation has been extended in an iterative fashion and shown to evaluate one dimensional nearly strongly singular integrals with a high degree of accuracy [5]. This paper presents an extension of the iterated sinh transformation to the evaluation of nearly strongly singular integrals arising in the solution of Laplace's equation in three dimensions.

If the distance $b$, between the source point and its projection onto the element of integration, is sufficiently large in comparison with the "diameter" (some measure of the size) of the element, say $b \geq 1$ for an element of diameter 1, then conventional Gaussian quadrature provides sufficient accuracy for evaluating such integrals $[2,20]$. However, smaller values of $b$ are of interest in many engineering applications of the boundary element method, such as 
those where the potential or flux must be calculated near the boundary. This occurs in the study of thin structures [17], sensitivity problems [23], contact problems [1] and displacement around open crack tips [4]. These applications involve complex geometries and, in addition, the numerical method used must be able to cope with the fact that the integrand becomes very large for small values of $b$.

Various methods have been proposed to deal with these integrals, such as dividing the element into an increasing number of subdivisions near to the source point [16], a singularity subtraction approach where the near singularity is subtracted out [3], use of polar coordinates [15], introduction of coordinate variable transformations to weaken the singularity before integration $[21,11,22]$ and combinations of the polar coordinates approach with variable transformations $[8,9,7,10,19,18]$.

As mentioned previously [14, 13], the sinh transformation automatically takes into account the distance from the source point to the position of the projection of the source point onto the element. The increased accuracy is achieved by the automatic clustering of integration points towards the nearly singular point. The iterated sinh transformation [5] clusters the integration points more strongly and is thus even more effective in evaluating strongly 
singular integrals.

Here, the iterated sinh transformation will be used (in a product fashion) to evaluate two dimensional nearly singular potential and flux integrals both on flat and curved elements and the results obtained will be compared with a variety of other techniques commonly used to evaluate these integrals.

\section{The Nearly Singular Integrals}

The nearly singular two dimensional integrals considered here arise in the three dimensional boundary element method and are of the general form [10]

$$
I=\int_{S} f(r) \phi d S
$$

where $\phi$ is some basis function, $S$ is (in general) a curved surface, $r=\left|\mathbf{x}-\mathbf{x}_{\mathbf{S}}\right|$

with $\mathbf{x}_{\mathbf{S}}$ the source point and $f(r)=g / r^{\alpha}$ for $\alpha=1,3$ or 5 where $g$ is a constant for potential integrals or a vector function for flux integrals. The integral $I$ becomes nearly singular when the distance $b$ between the source point $\mathbf{x}_{\mathbf{S}}$ and the surface $S$ becomes small. For $\alpha=1$, the integral is said to be nearly weakly singular and, for $\alpha=3$ or $\alpha=5$, it is said to be nearly strongly singular.

The usual practice is to transform the integral into an integration over 
the surface $-1 \leq s, t \leq 1$ (in a local coordinate system), resulting in the integral

$$
I_{i}=\int_{-1}^{1} \int_{-1}^{1} f(r(s, t)) \phi_{i}(s, t) J(s, t) d s d t
$$

where $\phi_{i}, i=1 \cdots 9$, is a bi-quadratic basis function as given in Table 1 and $J(s, t)$ is the Jacobian of the transformation of $S$ onto $[-1,1] \times[-1,1]$.

\subsection{Potential Integrals on a Flat Element}

The first integral considered in this paper, $I_{1, i}(\alpha)$, involves $f(r)=\frac{1}{r^{\alpha}}$ with $\alpha=3$ or 5 and is evaluated on the flat element $[-1,1] \times[-1,1]$. Here $J(s, t)=1$, $g=1$ and $b$ is the distance between the near-singular point and the point $\left(s_{0}, t_{0}\right)$ on the element closest to it so that $I_{1, i}(\alpha)$ is given by

$$
I_{1, i}(\alpha)=\int_{-1}^{1} \int_{-1}^{1} \frac{\phi_{i}(s, t)}{\left(\sqrt{\left(s-s_{0}\right)^{2}+\left(t-t_{0}\right)^{2}+b^{2}}\right)^{\alpha}} d s d t .
$$

Unfortunately, with these higher values of $\alpha$ it is not possible to obtain analytical values for these integrals, as it is with $\alpha=1$ [13].

\subsection{The Curved Element SPQ60}

Both potential and flux integrals will be evaluated on the curved surface SPQ60 [10], given by $x=\sin \psi \cos \phi, y=\sin \psi \sin \phi$ and $z=\cos \psi$ for 
$60^{\circ} \leq \psi \leq 120^{\circ}$ and $-30^{\circ} \leq \phi \leq 30^{\circ}$.

The patch SPQ60 is modelled by a 9-point Lagrangian element using the bi-quadratic basis functions given in Table 1. By defining $M=\frac{\sqrt{3}}{2}-1$, it is possible to write the three dimensional independent variables $(x, y, z)$ in terms of the local coordinates $(s, t)$ of the element as follows: $x(s, t)=$ $M\left(M s^{2} t^{2}+s^{2}+t^{2}\right)+1, y(s, t)=s\left(M t^{2}+1\right) / 2$ and $z(s, t)=\frac{t}{2}$. The Jacobian, $J(s, t)$, of the mapping from $(x, y, z)$ space to the local $(s, t)$ coordinate space is then given by $J(s, t)=\left(M t^{2}+1\right)\left(\frac{1}{16}+M^{2}\left[s^{2}+t^{2}\left(M s^{2}-1\right)^{2}\right]\right)^{\frac{1}{2}}$.

\subsection{Potential Integrals on a Curved Element}

The potential integrals on SPQ60 are given by

$$
I_{2 \mathrm{~A}, i}=\int_{\mathrm{SPQ} 60} \phi_{i} u^{*} d S
$$

and

$$
I_{2 \mathrm{~B}, i}=\int_{\mathrm{SPQ} 60} \phi_{i} q^{*} d S
$$

where $u^{*}=1 /(4 \pi r)$ and $q^{*}=\partial u^{*} / \partial \mathbf{x}_{\mathbf{S}}$. Here the distance $r$ is given by

$$
r=\sqrt{\left(x-x_{0}\right)^{2}+\left(y-y_{0}\right)^{2}+\left(z-z_{0}\right)^{2}},
$$

where $\mathbf{x}_{\mathbf{S}}=(x, y, z)$ is the source point and $\left(x_{0}, y_{0}, z_{0}\right)$ is the nearest point to $\mathbf{x}_{\mathbf{S}}$ on SPQ60, at a distance $b$ from it. 
Using the definitions from Section 2.2, it is found that

$$
I_{2 \mathrm{~A}, i}=\frac{1}{4 \pi} \int_{-1}^{1} \int_{-1}^{1} \frac{\phi_{i}(s, t) J(s, t)}{r(s, t)} d s d t
$$

for $r(s, t)=\left[\left(x(s, t)-x_{1}\right)^{2}+\left(y(s, t)-y_{1}\right)^{2}+\left(z(s, t)-z_{1}\right)^{2}\right]^{\frac{1}{2}}$, where $x_{1}=$ $k x_{0}, y_{1}=k y_{0}$ and $z_{1}=k z_{0}$, for $k=\left(r_{0}-b\right) / r_{0}$ where $r_{0}=\left[x_{0}^{2}+y_{0}^{2}+z_{0}^{2}\right]^{\frac{1}{2}}$.

The integral $I_{2 \mathrm{~A}, i}$ exhibits similar behaviour to the integral $I_{1, i}(1)$ (see equation (3)) and the integral $I_{2 \mathrm{~B}, i}$ exhibits similar behaviour to the integral $I_{1, i}(3)$

\subsection{Flux Integrals on a Curved Element}

The flux integrals are of the form

$$
I_{3 \mathrm{~A}, i}=\int_{\mathrm{SPQ} 60} \phi_{i} \frac{\partial u^{*}}{\partial \mathbf{x}_{\mathbf{S}}} d S
$$

and

$$
I_{3 \mathrm{~B}, i}=\int_{\mathrm{SPQ} 60} \phi_{i} \frac{\partial q^{*}}{\partial \mathbf{x}_{\mathbf{S}}} d S
$$

where

$$
\begin{gathered}
\frac{\partial u^{*}}{\partial \mathbf{x}_{\mathbf{S}}}=\frac{1}{4 \pi}\left(\frac{\rho \cos \theta}{r^{3}}, \frac{\rho \sin \theta}{r^{3}}, \frac{-b}{r^{3}}\right) \\
\frac{\partial q^{*}}{\partial \mathbf{x}_{\mathbf{S}}}=\frac{1}{4 \pi}\left(-3 b \frac{\rho \cos \theta}{r^{5}},-3 b \frac{\rho \sin \theta}{r^{5}},-\frac{1}{r^{3}}+\frac{3 b^{2}}{r^{5}}\right)
\end{gathered}
$$

for polar co-ordinates $(\rho, \theta)$ centred at $\left(x_{0}, y_{0}, z_{0}\right)$. 
The integral $I_{3 \mathrm{~A}, i}$ exhibits similar behaviour to the integral $I_{1, i}(3)$ and the integral $I_{3 \mathrm{~B}, i}$ exhibits similar behaviour to the integral $I_{1, i}(5)$.

\section{5 'Exact' values for the integrals}

Since the integrals described above are not amenable to analytic integration, 'exact' values for these integrals are found using the MATLAB (The MathWorks Inc.) numerical integration routine dblquad. The potential integrals $I_{2 \mathrm{~A}, i}$ and $I_{2 \mathrm{~B}, i}$ are evaluated with an accuracy of 15 decimal places, that is, $\mathrm{TOL}=1 \times 10^{-15}$. It is, however, necessary to apply a sinh transformation (as described in Section 3.1) to smooth the integral before evaluation with MATLAB in the cases where $b=0.001$ for $I_{2 \mathrm{~A}, i}$ and $b \leq 0.01$ for $I_{2 \mathrm{~B}, i}$.

The flux integrals $I_{3 \mathrm{~A}, i}$ and $I_{3 \mathrm{~B}, i}$ involve a stronger singularity than the potential integrals and even with the application of the sinh transformation it is possible to achieve 15 decimal place accuracy for the 'exact' solution only for $b \geq 0.3$, since TOL is dependent on the value of $b$. The maximum number of decimal places (indicated by the number in the parentheses) for which a solution could be obtained with dblquad over all 9 of the flux integrals involved in $I_{3 \mathrm{~A}, i}$ and $I_{3 \mathrm{~B}, i}$, are for $b=0.1(14) ; b=0.03(11) ; b=0.01(9) ; b=0.003$ (8); and $b=0.001$ (7). However, there are many individual cases where much 
greater accuracies can be achieved. These accuracies are sufficient since the 'exact' values are used here only to find Relative Errors just less than $10^{-6}$.

Finally, some of the integrals $I_{1, i}(\alpha)$ were obtained using MAPLE (Maplesoft, Waterloo Maple Inc.). For $\alpha=5$ it was possible to obtain values for $I_{1, i}(5)$ for $i=1,2, \ldots, 9$ with a working precision of 30 decimal places. However, for $I_{1, i}(3)$ it was possible to use MAPLE only for $i=2,4,6,8$. The remaining integrals were obtained using MATLAB as described above.

\section{Methods}

The numerical methods considered here, which are used to evaluate the nearsingular integrals $I_{i}$, given in equation (2), can be divided into two categories. The first type involves the application of a transformation (in each of the $s$ and $t$ directions), which smooths the integrand before Gaussian quadrature is applied. The sinh [13] and Telles [22] methods are of this type. The second type involves splitting the square, at the point on the square closest to the transformed source point, into (in general) four triangles and then introducing polar coordinates centred at that point. Next, various transfor-

mations are made, after which each triangle is mapped to $[-1,1] \times[-1,1]$ and 
then Gaussian quadrature is applied. The ' $\mathrm{L}$ ' methods involve application of both angular and radial transformations and these consist of the $\mathrm{L}_{2}[8]$, the $\log -\mathrm{L}_{1}[7]$ and the $\mathrm{L}_{1}^{-1 / 5}[10]$ methods. The polar method, which uses no transformations, will be simply designated 'polar'. The methods described above have also been summarised previously in [13].

\subsection{The iterated sinh transformation}

The iterated sinh transformation has been described previously for one dimensional integrals [5], as has the extension of the original sinh transformation [14] from one to two dimensions [13]. In two dimensions, two sinh transformations are required:

$$
s=s_{0}+b \sinh \left(\mu_{1}^{(0)} u-\eta_{1}^{(0)}\right)
$$

and

$$
t=t_{0}+b \sinh \left(\mu_{2}^{(0)} v-\eta_{2}^{(0)}\right) .
$$

Choosing $\mu_{1}^{(0)}, \mu_{2}^{(0)}, \eta_{1}^{(0)}$ and $\eta_{2}^{(0)}$, so that $[-1,1]$ maps onto [-1,1] for both $s$ and $t$, gives

$$
\mu_{1}^{(0)}=\frac{1}{2}\left[\operatorname{arcsinh}\left(\frac{1+s_{0}}{b}\right)+\operatorname{arcsinh}\left(\frac{1-s_{0}}{b}\right)\right]
$$


and

$$
\eta_{1}^{(0)}=\frac{1}{2}\left[\operatorname{arcsinh}\left(\frac{1+s_{0}}{b}\right)-\operatorname{arcsinh}\left(\frac{1-s_{0}}{b}\right)\right]
$$

with similar expressions for $\mu_{2}^{(0)}$ and $\eta_{2}^{(0)}$, where $s_{0}$ is replaced by $t_{0}$. These transformations include information about the nearly singular point $\left(s_{0}, t_{0}\right)$ as well as the distance $b$ from the source point to the element. Also, the mapping from $[-1,1]$ into $[-1,1]$ ensures that once the transformation has been made, Gaussian quadrature can be applied in the usual manner. It can be shown that from equation (6)

$$
r=\sqrt{\frac{b^{2}}{2}\left[\cosh \left(2\left(\mu_{1}^{(0)} u-\eta_{1}^{(0)}\right)\right)+\cosh \left(2\left(\mu_{2}^{(0)} v-\eta_{2}^{(0)}\right)\right)\right]},
$$

and all the integrals to be considered here possess a denominator having this form to a power of 3 or 5 . For example, the integral $I_{1, i}(\alpha)$ becomes

$$
\begin{aligned}
I_{1, i}(\alpha) & =\int_{-1}^{1} \int_{-1}^{1} \frac{\phi_{i}}{r^{\alpha}} d s d t \\
& =b^{2} \mu_{1}^{(0)} \mu_{2}^{(0)} \int_{-1}^{1} \int_{-1}^{1} \frac{\phi_{i}(s(u), t(v)) \cosh \left(\mu_{1}^{(0)} u-\eta_{1}^{(0)}\right) \cosh \left(\mu_{2}^{(0)} v-\eta_{2}^{(0)}\right)}{\left(\sqrt{\left.\left[\frac{b^{2}}{2}\left(\cosh \left(2\left(\mu_{1}^{(0)} u-\eta_{1}^{(0)}\right)\right)+\cosh \left(2\left(\mu_{2}^{(0)} v-\eta_{2}^{(0)}\right)\right)\right)\right]\right)^{\alpha}} d u d v .\right.}
\end{aligned}
$$

Hence, the integrands will have singularities when

$$
\cosh \left(2\left(\mu_{1}^{(0)} u-\eta_{1}^{(0)}\right)\right)+\cosh \left(2\left(\mu_{2}^{(0)} v-\eta_{2}^{(0)}\right)\right)=0
$$

Since, for real $x, \cosh x$ is always positive, the singularities must occur when

$$
\cosh \left(2\left(\mu_{1}^{(0)} u-\eta_{1}^{(0)}\right)\right)=0 \quad \text { and } \quad \cosh \left(2\left(\mu_{2}^{(0)} v-\eta_{2}^{(0)}\right)\right)=0
$$


as $u$ and $v$ are independent.

In the complex plane, there are infinitely many solutions to these equations, but the nearest singularities to the interval $[-1,1]$ are at $z_{1}, \overline{z_{1}}$ and $z_{2}$, $\overline{z_{2}}$, where

$$
z_{1}:=a_{1}+i b_{1} \quad \text { and } \quad z_{2}:=a_{2}+i b_{2}
$$

with

$$
a_{1}=\frac{\eta_{1}^{(0)}}{\mu_{1}^{(0)}}, \quad b_{1}=\frac{\pi}{4 \mu_{1}^{(0)}}, \quad a_{2}=\frac{\eta_{2}^{(0)}}{\mu_{2}^{(0)}}, \quad b_{2}=\frac{\pi}{4 \mu_{2}^{(0)}} .
$$

Based on the one dimensional iterated sinh transformation it is reasonable to apply the sinh transformation again and define an iterated sinh transformation by letting

$$
u=a_{1}+b_{1} \sinh \left(\mu_{1}^{(1)} p-\eta_{1}^{(1)}\right)
$$

and

$$
v=a_{2}+b_{2} \sinh \left(\mu_{2}^{(1)} q-\eta_{2}^{(1)}\right)
$$

where

$$
\mu_{1}^{(1)}=\frac{1}{2}\left[\operatorname{arcsinh}\left(\frac{1+a_{1}}{b_{1}}\right)+\operatorname{arcsinh}\left(\frac{1-a_{1}}{b_{1}}\right)\right]
$$

and

$$
\eta_{1}^{(1)}=\frac{1}{2}\left[\operatorname{arcsinh}\left(\frac{1+a_{1}}{b_{1}}\right)-\operatorname{arcsinh}\left(\frac{1-a_{1}}{b_{1}}\right)\right]
$$


with similar expressions for $\mu_{2}^{(1)}$ and $\eta_{2}^{(1)}$ where $a_{1}$ and $b_{1}$ are replaced by $a_{2}$ and $b_{2}$, respectively. After the second sinh transformation, the integral $I_{1, i}(\alpha)$ is now

$$
\begin{aligned}
I_{1, i}(\alpha)= & \frac{b^{2} \pi^{2} \mu_{1}^{(1)} \mu_{2}^{(1)}}{16} \int_{-1}^{1} \int_{-1}^{1} \phi_{i}(s(u(p)), t(v(q))) \times \\
& \frac{\cosh \left(\frac{\pi}{4} \sinh \left(\mu_{1}^{(1)} p-\eta_{1}^{(1)}\right)\right) \cosh \left(\frac{\pi}{4} \sinh \left(\mu_{2}^{(1)} q-\eta_{2}^{(1)}\right)\right) \cosh \left(\mu_{1}^{(1)} p-\eta_{1}^{(1)}\right) \cosh \left(\mu_{2}^{(1)} q-\eta_{2}^{(1)}\right)}{\left(\sqrt{\left[\frac{b^{2}}{2}\left(\cosh \left(\frac{\pi}{2} \sinh \left(\mu_{1}^{(1)} p-\eta_{1}^{(1)}\right)\right)+\cosh \left(\frac{\pi}{2} \sinh \left(\mu_{2}^{(1)} p-\eta_{2}^{(1)}\right)\right)\right]\right.}\right)^{\alpha}} d q d p .
\end{aligned}
$$

From the point of view of numerical integration, this second transformation has the effect of clustering the integration points nearer to the singularity and hence better capturing the spiked behaviour of the nearly singular integrand.

The original sinh transformation, as proposed in [13] and resulting in equation (17) will, from now on, be referred to as "one iteration" of the sinh transformation. The second sinh transformation, resulting in equation (23), will be referred to as "two iterations" of the sinh transformation. Finally, untransformed Gaussian quadrature will be referred to as "zero iterations" of the sinh transformation. 


\subsection{Relative Error}

The techniques described above are compared in terms of the Relative Error, defined by

$$
\text { Relative Error }=\left|\frac{I_{\text {approximate }}-I_{\text {exact }}}{I_{\text {exact }}}\right|,
$$

where $I_{\text {approximate }}$ and $I_{\text {exact }}$ are the approximate and 'exact' values, respec-

tively, of the integral under consideration. The integral $I_{\text {exact }}$ is evaluated using MATLAB or MAPLE, as discussed in Section 2.5.

\section{Results}

\subsection{Example 1}

Integrals of the form $I_{1, i}(\alpha)$ (equation (3)) are evaluated using the Telles, Log- $\mathrm{L}_{1}$ and polar methods as well as with zero (that is, straight Gaussian quadrature), one and two iterations of the sinh transformation, as detailed in the previous section. Values of Relative Error are found for each of these methods, where the number of Gaussian integration points, NP, is the same in both the $s$ and $t$ directions. The integrals are evaluated for each basis function $\phi_{1}$ to $\phi_{9}$, for values of $s_{0}$ and $t_{0}$ in $[0,1)$, for $b=0.1,0.01,0.001,0.0001$ and 0.00001 with $\alpha=3$ and $\alpha=5$. Since the $\log -\mathrm{L}_{1}$ and polar methods require 
splitting the square into four triangles each requiring Gaussian quadrature, these two methods are compared using $\mathrm{NP} / 2 \times \mathrm{NP} / 2$ integration points in each subregion (note that it is assumed that NP is even).

Example plots are shown in Figure 1, where Relative Error is plotted against $s_{0} \in[0,0.9]$ for values of $t_{0}$ each 0.1 from 0 to 0.9 . In each case $b=0.0001, \alpha=3$ (left column) and $\alpha=5$ (right column) and basis function $\phi_{5}$ is used. Results using one and two iterations of the sinh transformation were obtained with $\mathrm{NP}=20$ and the results for the $\log -\mathrm{L}_{1}$ method were obtained with $\mathrm{NP}=10$. A comparison of the six graphs shows that the Relative Error for the $\log -\mathrm{L}_{1}$ method is generally lower than for one iteration of the sinh transformation. However, after two iterations of the sinh transformation Relative Errors are at least one order of magnitude smaller than either of the other two methods for $\alpha=3$ and two orders of magnitude smaller for $\alpha=5$. Interestingly, the Log- $\mathrm{L}_{1}$ method shows the smoothest variation of Relative Error with $s_{0}$ of the three methods shown.

In order to quantify these differences, the data are summarised using a geometric mean $\bar{g}$ and a geometric standard deviation $\sigma_{g}$, via the notation $\bar{g} \stackrel{\times}{\div} \sigma_{g}[12,13]$, as the Relative Error is best presented on a log scale. These quantities are calculated so that the two values reflect the ability of a 
particular method to approximate $I_{1, i}(\alpha)$ over all the nearly singular points $\left(s_{0}, t_{0}\right) \in[0,1) \times[0,1)$.

Relative Errors are calculated for particular values of $b$ and NP for each basis function at each 0.1 increment for both $s_{0}$ and $t_{0}$ in $[0,1)$ (i.e., 100 points). Due to the symmetry of the basis functions, $\phi_{2}(s, t)=\phi_{4}(t, s)$, $\phi_{3}(s, t)=\phi_{7}(t, s)$ and $\phi_{6}(s, t)=\phi_{8}(t, s)$, the means and standard deviations of the Relative Errors over $[0,1) \times[0,1)$ will be the same for these pairs of basis functions.

Table 2 summarises the geometric mean and standard deviation of the Relative Error in evaluating integral $I_{1, i}(3)$, for each basis function with $\left(s_{0}, t_{0}\right) \in[0,1) \times[0,1)$ and over all basis functions, with $b=0.0001$. The number of points NP, used for the Gaussian quadrature, is indicated after the method name. Table 3 summarises the same information for the integral $I_{1, i}(5)$.

From Table 2 it can be seen that the geometric means of the Relative Errors for one and two iterations of the sinh transformation and the Log$\mathrm{L}_{1}$ transformation for the integral $I_{1,5}(3)$, plotted in Figure 1 (a), (c) and (e), are $4.0 \times 10^{-3}, 1.1 \times 10^{-4}$ and $5.9 \times 10^{-3}$, respectively, indicating that two iterations of the sinh transformation are overall about 40 times more 
accurate than one iteration of the sinh transformation and about 60 times more accurate than the $\log -\mathrm{L}_{1}$ transformation. Also, from Table 3, the geometric means of the Relative Error for the same methods applied to the integral $I_{1,5}(5)$, from Figure 1 (b), (d) and (f), are $7.2 \times 10^{-2}, 1.2 \times 10^{-5}$ and $3.3 \times 10^{-2}$, respectively. In this case, two iterations of the sinh transformation give Relative Errors three orders of magnitude lower than the other two cases. Interestingly, for two iterations of the sinh transformation, the Relative Errors for $I_{1, i}(5)$ are lower than those for the integral $I_{1, i}(3)$ for each basis function.

Tables 4 and 5 summarise the Relative Error over all basis functions and all nearly singular points at various values of $b$ for the integrals $I_{1, i}(3)$ and $I_{1, i}(5)$, respectively, with $N P=20$ or 10 , as indicated. It can be seen in both cases that using two iterations of the sinh transformation is clearly superior to any other choice of the integration technique. Again, this transformation is more accurate for $I_{1, i}(5)$ than for $I_{1, i}(3)$.

Finally, Figure 2 shows the Relative Error plotted against $b$ at the point $\left(s_{0}, t_{0}\right)=(0.5,0.5)$ for each of the six methods with basis function $\phi_{5}$ and NP $=20$ or 10, as indicated, for $\alpha=3$ (Figure 2(a)) and $\alpha=5$ (Figure 2(b)). It can easily be seen that two iterations of the sinh transformation yields the 
most accurate evaluation of the integrals. It is also the case that the smallest errors are obtained with $\alpha=5$.

\subsection{Example 2}

As a second example, integrals of the form $I_{2 \mathrm{~A}, i}(4), I_{2 \mathrm{~B}, i}(5), I_{3 \mathrm{~A}, i}(8)$ and $I_{3 \mathrm{~B}, i}$ (9) are considered. The integrals $I_{2 A, i}$ correspond to $\alpha=1$, the integrals $I_{2 B, i}$ and $I_{3 \mathrm{~A}, i}$ correspond to $\alpha=3$ and the integrals $I_{3 \mathrm{~B}, i}$ correspond to $\alpha=5$. All integrals are evaluated using one or two iterations of the sinh transformation as well as the Telles, $\log -\mathrm{L}_{1}$ and $L_{1}^{-1 / 5}$ methods. Straightforward Gaussian quadrature (zero iterations) was not considered since, for these types of integrals, large numbers of Gaussian points are required, especially for small values of $b$.

Table 6 summarises the Relative Errors for the integral $I_{3 \mathrm{~A}, i}$ of the particular form

$$
I_{3 \mathrm{~A}, i, x}=\int_{\mathrm{SPQ} 60} \phi_{i} \frac{\partial u^{*}}{\partial x} d S .
$$

The integrals are evaluated using one and two iterations of the sinh transformation, as well as the Telles, Log- $\mathrm{L}_{1}$ and $\mathrm{L}^{-1 / 5}$ methods for $\mathrm{NP}=10$ and 20 integration points. The results show that for $\mathrm{NP}=10$, one iteration of the sinh transformation is generally sufficient, except for $b=0.001$ when the $\mathrm{L}^{-1 / 5}$ 
transformation is superior. However, for $\mathrm{NP}=20$, two iterations of the sinh transformation provide the most accurate evaluation of the integrals, often by one order of magnitude or more. Note that since both of the integrals $I_{2 \mathrm{~B}, i}$ and $I_{3 \mathrm{~A}, i, x}$ behave like a nearly strongly singular integral with $\alpha=3$, only results for the particular integral $I_{3 \mathrm{~A}, i, x}$ have been shown. Similar results and comments apply to the integral $I_{2 \mathrm{~B}, i}$.

Table 7 summarises a similar set of integral evaluations, this time for the integral

$$
I_{3 \mathrm{~B}, i, x}=\int_{\mathrm{SPQ} 60} \phi_{i} \frac{\partial q^{*}}{\partial x} d S .
$$

Recall that this integral behaves like a strongly singular integral with $\alpha=5$. For this integral one iteration of the sinh transformation yields the most accurate evaluation for $\mathrm{NP}=10$, followed by two iterations of the sinh transformation. However, when $\mathrm{NP}=20$, two iterations of the sinh transformation yield Relative Errors up to two orders of magnitude smaller than the $\mathrm{L}^{-1 / 5}$ transformation and three orders of magnitude smaller than one iteration of the sinh transformation and the $\log -\mathrm{L}_{1}$ transformation.

For the sake of completeness, Table 8 summarises the Relative Errors for evaluating the integral $I_{2 \mathrm{~A}, 1}$ for $N P=20$ or 10 , as indicated. The table shows that one iteration of the sinh transformation is sufficient and using 
two iterations actually decreases the accuracy of the approximate values dramatically as $b$ becomes smaller. In fact, for this integral, one iteration of the sinh transformation produces the most accurate approximations to the values of the integrals for the methods considered.

\subsection{Comparison of Computational Efficiency}

Table 9 compares the computational efficiency of the various methods in evaluating both the potential integral $I_{2 \mathrm{~A}, 1}$ and the flux integral $I_{3 \mathrm{~B}, 1, x}$ on the curved element SPQ60. These integrals were evaluated for a range of $b$ values using the minimum number of points required to produce a relative error of less than $10^{-6}$ (as presented previously [13]). It was found that, for a MacBook Pro laptop with a 2.6 GHz Intel Core i7 processor, the time taken to evaluate any particular integral is of the order of tens of microseconds or less. Hence, each integral was evaluated 10000 times and the CPU times required to evaluate the potential and flux integrals mentioned above are presented in Table 9.

For $b \leq 0.1$, the time taken by all the methods to evaluate one integral is between 10-20 microseconds, excluding Telles where large numbers of points are required for small $b$ values. Times of up to 35 microseconds per integral 
are found for both iterations of the sinh transformation and the $\mathrm{L}_{1}^{-1 / 5}$ method for $b=0.003$ or 0.001 . However, for the flux integral $I_{3 \mathrm{~B}, 1}$, two iterations of the sinh transformation are evaluated more quickly than one iteration to achieve the same level of accuracy. This reflects the fact that the iterated sinh transformation is best suited to strongly nearly-singular integrals at small values of $b$.

\section{Discussion}

This work presents a number of methods that can be used to evaluate the nearly singular integrals which arise in the three dimensional boundary element method, when the source point is close to, but not on, the element of integration. In every case, the original (curved) element is mapped first to $[-1,1] \times[-1,1]$. All the methods (excluding conventional Gaussian quadrature and the polar method) use transformations of the variables involved in the integration. The Telles and iterative sinh methods differ from the other methods in that the transformations are applied in a simple product fashion to the integral, whereas the other methods are based on the polar method, which breaks the square at the point $\mathbf{x}_{\mathbf{S}}$, into (in general) four triangles. 
These triangles are then mapped into $[-1,1] \times[-1,1]$ and transformations are applied to the angular and radial variables in each of the triangles.

Two types of examples are considered here: integrals on a flat element with large inverse powers of the radial variable and both potential and flux integrals on a curved element. For the integrals on a flat element, Relative Errors are calculated across 100 points in $[0,1) \times[0,1)$, for each basis function. A summary of these results is presented in Tables 2, 3, 4 and 5, where the geometric mean and standard deviation are given, for various values of $b$ and NP over all basis functions. Calculations for the methods based on the polar method are performed using $\mathrm{NP} / 2 \times \mathrm{NP} / 2$ points, as indicated in brackets beside the method name, because these methods use four triangles and hence four times as many points (for non-edge source points) as do the other methods.

Tables 2 and 3 show that for the strongly singular integrals $I_{1, i}(3)$ and $I_{1, i}(5)$ two iterations of the sinh transformation produce superior results over all basis function for the given values of $b$. Further, as summarised in Tables 4 and 5, two iterations give the more accurate results averaged, in a geometrical sense, over all basis functions. The same observation applies for all values of $b$ considered for 20 Gaussian integration points. The reason for the superior 
performance of two iterations of the sinh transformation lies in the fact that this transformation clusters the integration points more strongly towards the nearly singular point. Strongly singular integrals are characterised by an "almost" Dirac-delta function-like behaviour with the integrand becoming very large over a small subregion of the region of integration and moreor-less zero elsewhere. Hence, to capture accurately this behaviour, more integration points are required close to the nearly singular point.

For weakly singular integrals of the form discussed in [13] (i.e. with $\alpha=1$ ) it has already been shown that generally one iteration of the sinh transformation yields very accurate evaluations. It has also been shown previously that one iteration of the sinh transformation is superior to two iterations for nearly weakly singular one dimensional integrals [5]. A possible reason for this is shown in Figure 3.

Figure 3 shows the position of the integration points (marked by ' + ' signs) for zero, one and two iterations of the sinh transformation and contour lines for the function

$$
f(x, y)=\frac{\phi_{5}(x, y)}{\left((x-0.2)^{2}+(y-0.2)^{2}+0.01\right)^{\alpha / 2}}
$$

for $\alpha=1$ (a nearly weakly singular integral, left hand column) and $\alpha=3$ (a nearly strongly singular integral, right hand column). Each subfigure shows 
the maximum value of the function $f(x, y)$ and its associated contours, spaced at $10 \%$ of this maximum value. From Figures $3(\mathrm{a})$ and $3(\mathrm{~b})$ it can be seen that there are very few integration points near the peak of the function $f$ for zero iterations of the sinh transformation. In Figures 3(c) and 3(d), with one iteration of the sinh transformation, more integration points have moved under the peak of the function $f$. Hence, one iteration of the sinh transformation evaluates the integral more accurately. Finally, Figures 3(e) and $3(\mathrm{f})$ show that two iterations bring even more integration points under the peak of $f$. This is good for $\alpha=3$ (Figure 3(f)) as more points are located where the function takes on larger values. However, it is not so good when $\alpha=1$ (Figure $3(\mathrm{e})$ ). Here there are too few points located outside the $10 \%$ contour to adequately sample the function at smaller function values, thus decreasing the accuracy of the integration procedure.

This behaviour is further borne out in Table 8 which summarises the evaluation of the integral $I_{2 \mathrm{~A}, 1}$ over the curved element SPQ60. The nearly weakly singular integral is evaluated most accurately by one iteration of the sinh transformation. Application of a further iteration only reduces this accuracy. For small values of $b$, it is interesting to note (although it is not shown here) that doubling the number of integration points from 10 to 20 
in each coordinate direction improves the accuracy by about three orders of magnitude for all transformation methods, except the Telles and $\mathrm{L}^{-1 / 5}$ transformations. Given the results previously presented in [13], integrals of the form $I_{1, i}(1)$ were not considered here as one iteration of the sinh transformation yields more accurate results than two iterations.

From Table 6 it is observed that, when using 10 Gaussian integration points, the Log- $\mathrm{L}_{1}, \mathrm{~L}^{-1 / 5}$ (both with 5 integration points) and one iteration of the sinh transformation give similar levels of accuracy when evaluating the integral $I_{3 \mathrm{~A}, 1, x}$ (an integral with a near singularity of order $\alpha=3$ ) for all $b$ values considered. For larger $b$, two iterations of the sinh transformation generally perform worse or no better than one iteration. However, when the number of integration points is doubled in each coordinate direction, using two iterations of the sinh transformation decreases the Relative Error by about two orders of magnitude, whereas the other methods show little improvement, especially at small $b$ values. The reasons for this are again due to the placement of the integration points with the different transformations.

Similar observations can be made for the integral $I_{3 \mathrm{~B}, i, x}$ (an integral with a near singularity of order $\alpha=5$ ) (Table 7); however, when doubling the number of integration points, the accuracy of two iterations of the sinh trans- 
formation is improved by up to four orders of magnitude. Improvements in the other methods are also observed, especially at larger $b$ values, but not at the more important small values of $b$.

A consequence of the above observation is also borne out in Table 9 . For the more strongly nearly-singular integral $I_{3 \mathrm{~B}, i, x}$, computation times are less than those required for the integral $I_{2 \mathrm{~A}, i}$ with two iterations of the sinh transformation. Also, one iteration of the sinh transformation requires more CPU time to evaluate the integral $I_{3 \mathrm{~B}, i, x}$ than two iterations of the sinh transformation.

\section{Conclusions}

This paper has presented a comparison of a number of different methods for evaluating the nearly strongly singular flux integrals which must be calculated as part of an implementation of the three dimensional boundary element method. These integrals become nearly singular when the distance $b$, between the source point and the element over which the integration is performed, becomes small in relation to the size of the element. Nearly weakly singular potential integrals were also considered for the sake of completeness. 
It has been shown that when $b \geq 1$ either one iteration of the sinh transformation or the Telles method works effectively for the nearly strongly singular integrals. As the value of $b$ decreases it is generally the case that one or two iterations of the sinh transformation yield the most accurate evaluations of the integrals. Further, when the number of integration points is doubled, errors obtained using two iterations of the sinh transformation are further reduced, whereas error levels for the other methods remain similar. However, for the nearly weakly singular integrals, one iteration of the sinh transformation is superior to two iterations.

Final conclusions are therefore that, given its ease of implementation and its ability to produce very low Relative Errors across the full range of values of $b$ for both potential and flux integrals, the sinh transformation method should be used to evaluate all nearly singular integrals. For the nearly weakly singular integrals, one iteration is sufficient but for the nearly strongly singular integrals, two iterations are required. Lastly, for small values of $b$ it is recommended that a larger number (say 20) Gaussian points be used with two iterations of the sinh transformation.

Studies of the form presented here raise questions related to error estimates. So far the authors have published error estimates for two dimensional 
Gaussian quadrature (zero iterations of the sinh transformation) [6]. Error estimates for the iterated sinh transformation for two dimensional integrals is an area of ongoing research. 


\section{References}

[1] M. H. Aliabadi, D. Martin, Boundary element hyper-singular formulation for elastoplastic contact problems, International Journal for $\mathrm{Nu}$ merical Methods in Engineering 48 (2000) 995-1014.

[2] C. A. Brebbia, J. C. F. Telles, L. C. Wrobel, Boundary Element Techniques, Springer-Verlag, Berlin, 1984.

[3] T. A. Cruse, R. Aithal, Non-singular boundary integral equation implementation, International Journal for Numerical Methods in Engineering 36 (1993) 237-254.

[4] T. Dirgantara, M. H. Aliabadi, Crack growth analysis of plates loaded by bending and tension using dual boundary element method, International Journal of Fracture 105 (2000) 27-74.

[5] D. Elliott, P. R. Johnston, The iterated sinh transformation, International Journal for Numerical Methods in Engineering 75 (1) (2008) 4357.

[6] D. Elliott, P. R. Johnston, B. M. Johnston, Estimates of the error in Gauss-Legendre quadrature for double integrals, Journal of Computa- 
tional and Applied Mathematics 236 (2011) 1552-1561.

[7] K. Hayami, A robust numerical integration method for three dimensional boundary element analysis, in: M. Tanaka, C. A. Brebbia, T. Honma (eds.), Boundary Elements XII, Springer-Verlag, 1990, pp. 33-51.

[8] K. Hayami, C. A. Brebbia, A new coordinate transformation method for singular and nearly singular integrals over general curved boundary elements, in: C. A. Brebbia, W. L. Wendland, G. Kuhn (eds.), Boundary Elements IX, Springer-Verlag, 1987, pp. 375-399.

[9] K. Hayami, C. A. Brebbia, Quadrature methods for singular and nearly singular integrals in 3-D boundary element method, in: C. A. Brebbia (ed.), Boundary Elements X, Springer-Verlag, 1988, pp. 237-264.

[10] K. Hayami, H. Matsumoto, A numerical quadrature for nearly singular boundary element integrals, Engineering Analysis with Boundary Elements 13 (1994) 143-154.

[11] T. Higashimachi, N. Okamoto, Y. Ezawa, T. Aizawa, A. Ito, Interactive structural analysis system using the advanced boundary element method, in: C. Brebbia, T. Futagami, M. Tanaka (eds.), Boundary Elements - Proc. 5th Inst. Conf., Springer-Verlag, 1983. 
[12] B. M. Johnston, P. R. Johnston, A comparison of transformation methods for evaluating two dimensional weakly singular integrals, International Journal for Numerical Methods in Engineering 56 (4) (2003) 589607.

[13] B. M. Johnston, P. R. Johnston, D. Elliott, A sinh transformation for evaluating two dimensional nearly singular boundary element integrals, International Journal for Numerical Methods in Engineering 69 (7) (2007) 1460-1479.

[14] P. R. Johnston, D. Elliott, A sinh transformation for evaluating nearly singular boundary element integrals, International Journal for Numerical Methods in Engineering 62 (4) (2005) 564-578.

[15] M. Koizumi, M. Utamura, A polar coordinate integration scheme with a hierarchical correction procedure to improve accuracy, in: T. A. Cruse (ed.), Proc. IUTAM Symp. on Advanced Boundary Element Methods, 1987, pp. 215-222.

[16] J. C. Lachat, J. O. Watson, Effective numerical treatment of boundary integral equations: A formulation for three-dimensional electrostatics, 
International Journal for Numerical Methods in Engineering 10 (1976) $273-289$.

[17] Y. J. Liu, Analysis of shell-like structures by the boundary element method based on 3-D elasticity: formulation and verification, International Journal for Numerical Methods in Engineering 41 (1998) 541-558.

[18] L. Scuderi, On the computation of nearly singular integrals in 3D BEM collocation, International Journal for Numerical Methods in Engineering 74 (11) (2008) 1733-1770.

[19] V. Sladek, J. Sladek, M. Tanaka, Evaluation of $1 / r$ integrals in BEM formulations for 3-D problems using coordinate multitransformations, Engineering Analysis with Boundary Elements 20 (1997) 229-244.

[20] A. H. Stroud, D. Secrest, Gaussian Quadrature Formulas, Prentice-Hall, Englewood Cliffs, N.J., 1966.

[21] M. Takahashi, M. Mori, Quadrature formulas obtained by variable transformation, Numer. Math. 21 (1973) 206-219. 
[22] J. C. F. Telles, A self-adaptive co-ordinate transformation for efficient numerical evaluation of general boundary element integrals, International Journal for Numerical Methods in Engineering 24 (1987) 959-973.

[23] D. Zhang, F. J. Rizzo, Y. J. Rudolphi, Stress intensity sensitivities via hypersingular boundary element integral equations, Computational Mechanics 23 (1999) 389-396. 


\section{Captions}

Table 1: The nine bi-quadratic basis functions.

Table 2: Table of geometric means and standard deviations of the Relative Error for the integral $I_{1, i}(3)$ for $b=0.0001$, for each basis function and evaluation method, using 10 or 20 Gaussian integration points as indicated. Table 3: Table of geometric means and standard deviations of the Relative Error for the integral $I_{1, i}(5)$ for $b=0.0001$, for each basis function and evaluation method, using 10 or 20 Gaussian integration points as indicated. Table 4: Table of geometric means and standard deviations of the Relative Error for the integral $I_{1, i}(3)$ for 10 or 20 Gaussian integration points as indicated, over all basis functions, for various values of $b$, for each evaluation method.

Table 5: Table of geometric means and standard deviations of the Relative Error for the integral $I_{1, i}(5)$ for 10 or 20 Gaussian integration points as indicated, over all basis functions, for various values of $b$, for each evaluation method.

Table 6: Table of Relative Errors for the basis function $\phi_{1}$ found when evaluating the integral $I_{3 \mathrm{~A}, 1, x}$ on the curved element SPQ60, for a range of $b$ values and a number of different evaluation methods with 10 or 20 Gaussian 
integration points as indicated.

Table 7: Table of Relative Errors for the basis function $\phi_{1}$ found when evaluating the integral $I_{3 \mathrm{~B}, 1, x}$ on the curved element SPQ60, for a range of $b$ values and a number of different evaluation methods with 10 or 20 Gaussian integration points as indicated.

Table 8: Table of Relative Errors for the basis function $\phi_{1}$ found when evaluating the integral $I_{2 \mathrm{~A}, 1}$ on the curved element SPQ60, for a range of $b$ values and a number of different evaluation methods.

Table 9: Table of the times (in seconds) to make 10000 evaluations of the potential integral $I_{2 \mathrm{~A}, 1}$ and the flux integral $I_{3 \mathrm{~A}, 1, x}$, on the curved element SPQ60, for various methods and for various values of $b$.

Figure 1: Plot of Relative Error as $s_{0}$ varies between 0 and 0.9 for values of $t_{0}$ between 0 and 0.9 in steps of 0.1 for the indicated solution methods with $\mathrm{NP}=20$ in (a), (b), (c) and (d) and $\mathrm{NP}=10$ in (e) and (f) and $b=0.0001$ using the basis function $\phi_{5}$ in the evaluation of the integrals $I_{1,5}(3)$ (left hand column) and $I_{1,5}(5)$ (right hand column).

Figure 2: Plot of Relative Error versus $b$ for various methods for evaluating the integrals (a) $I_{1,5}(3)$ and (b) $I_{1,5}(5)$ at the point $(0.5,0.5)$ with $\mathrm{NP}=10$ or 20 as indicated. 
Figure 3: Combined plot of Gaussian integration points ('+') and contours of the function $f(x, y)$, given by equation (27), for various numbers of iterations of the sinh transformation and for nearly weakly singular $(\alpha=1)$ and nearly strongly singular $(\alpha=3)$ integrals. The level of the outermost contour is $10 \%$ of the maximum value of the function. 


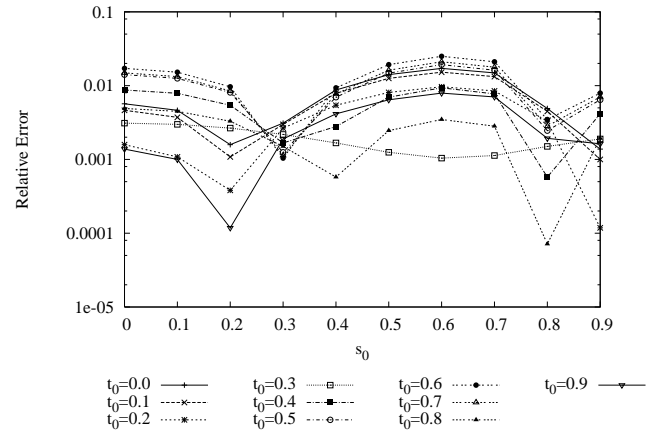

(a) Iteration $1(\alpha=3)$

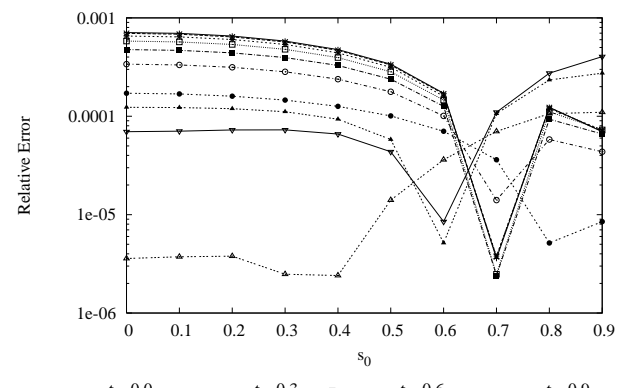

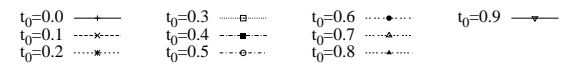

(c) Iteration $2(\alpha=3)$

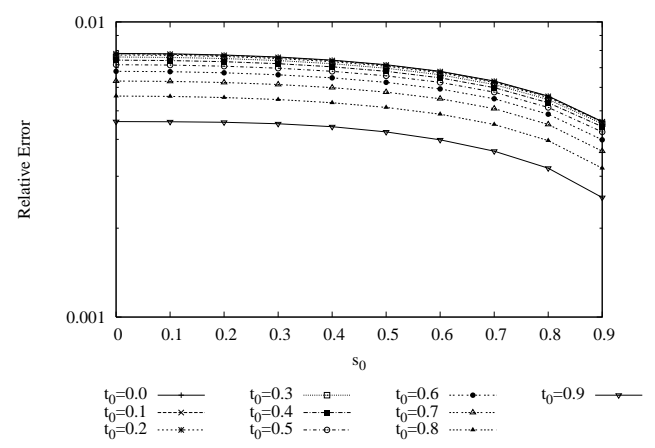

(e) Log-L $\mathrm{L}_{1}$ Method $(\alpha=3)$

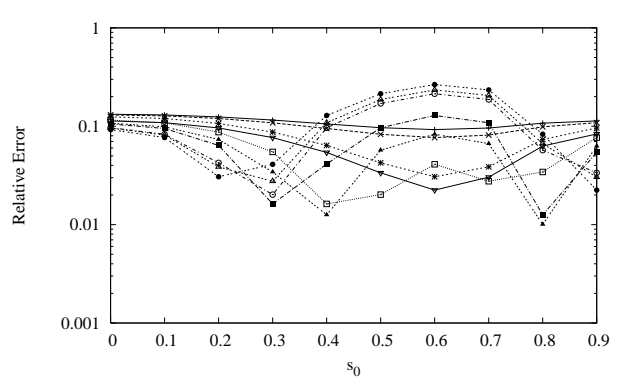

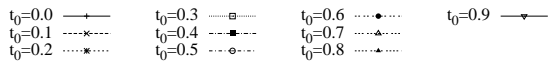

(b) Iteration $1(\alpha=5)$

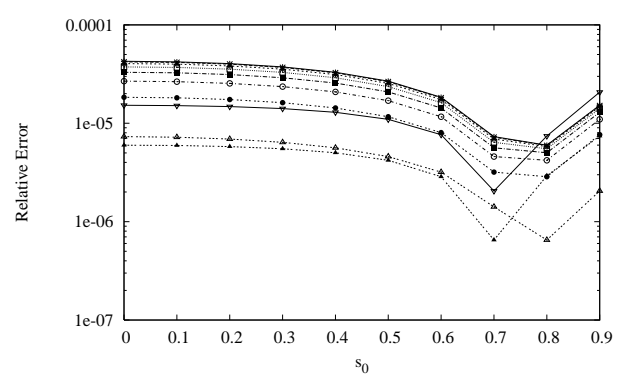

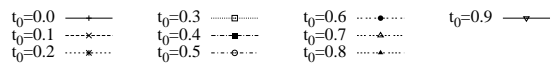

(d) Iteration $2(\alpha=5)$

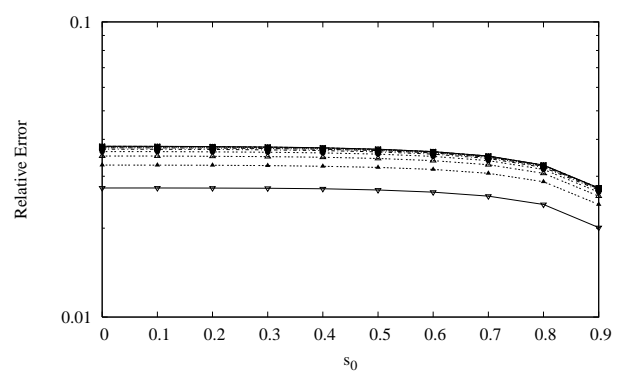

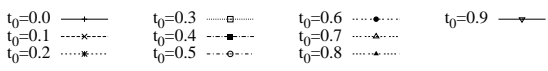

(f) Log- $\mathrm{L}_{1}$ Method $(\alpha=5)$

Figure 1: 


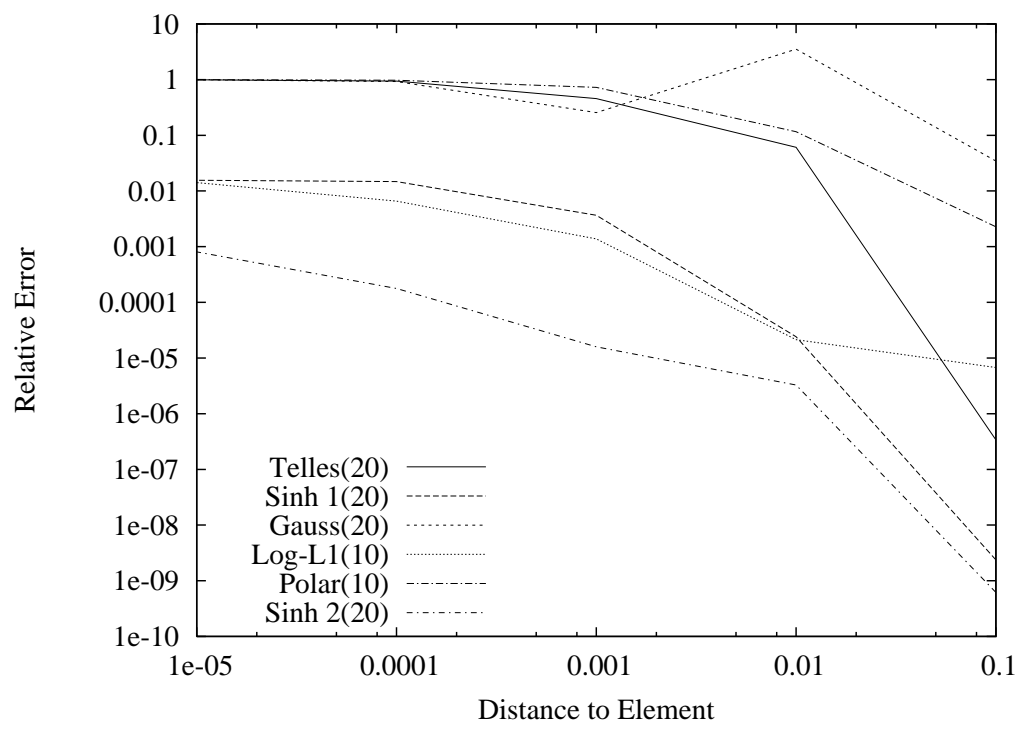

(a) $\alpha=3$

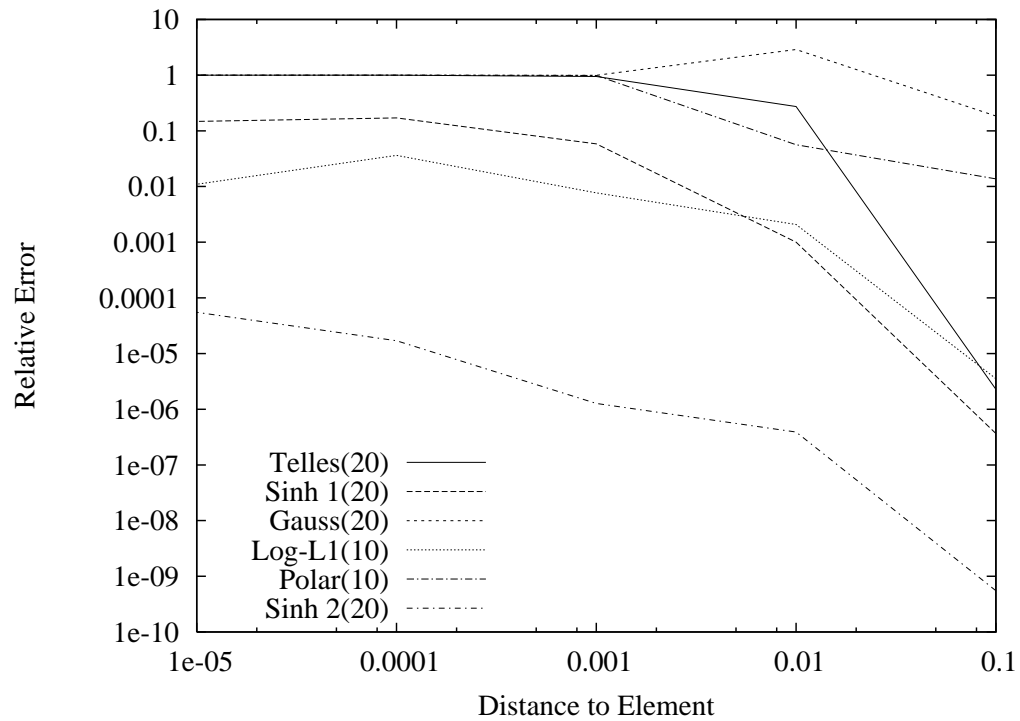

(b) $\alpha=5$

Figure 2: 


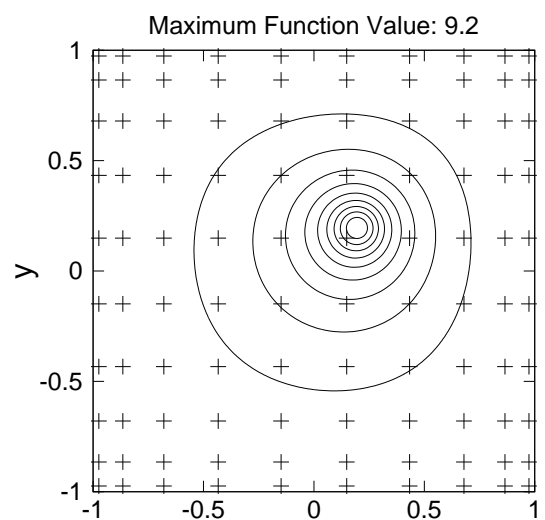

(a) Iteratioñ $0(\alpha=1)$

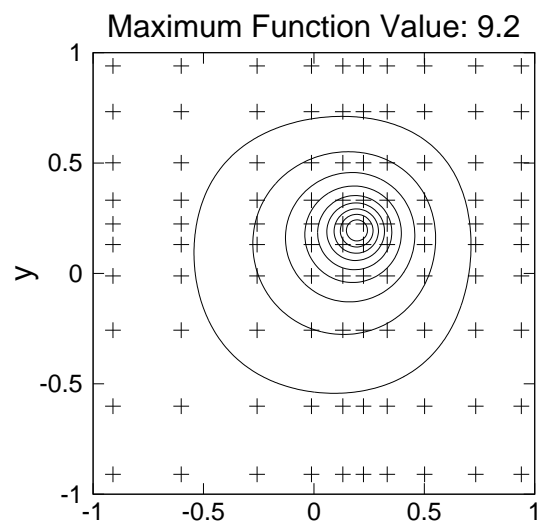

(c) Iteration $1(\alpha=1)$

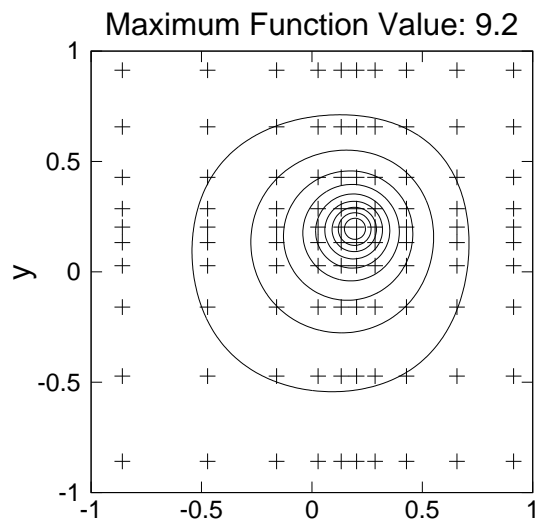

(e) Iteration $2(\alpha=1)$

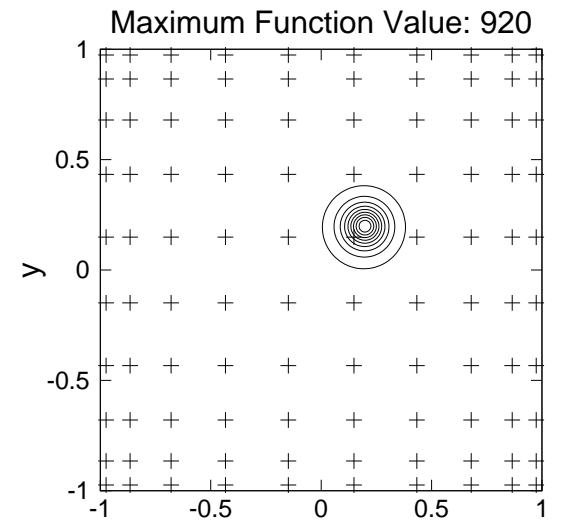

(b) Iteratioû $0(\alpha=3)$

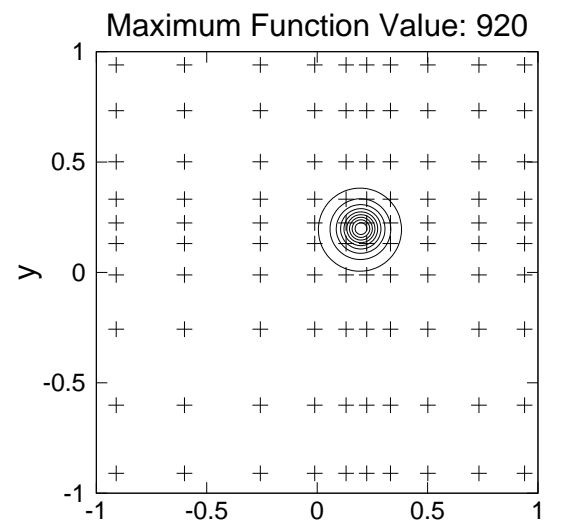

(d) Iteration $1(\alpha=3)$

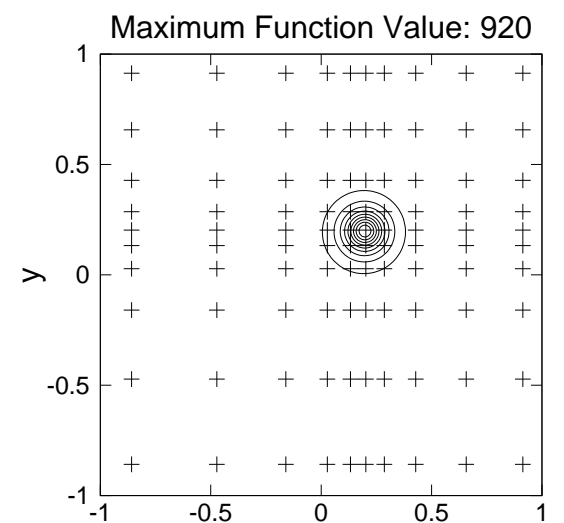

(f) Iteration $2(\alpha=3)$

Figure 3: 


\begin{tabular}{|c|c|}
\hline$i$ & $\phi_{i}(s, t)$ \\
\hline 1 & $\frac{1}{4} s(s-1) t(t-1)$ \\
2 & $\frac{1}{2}\left(1-s^{2}\right) t(t-1)$ \\
3 & $\frac{1}{4} s(s+1) t(t-1)$ \\
4 & $\frac{1}{2} s(s-1)\left(1-t^{2}\right)$ \\
5 & $\left(1-s^{2}\right)\left(1-t^{2}\right)$ \\
6 & $\frac{1}{2} s(s+1)\left(1-t^{2}\right)$ \\
7 & $\frac{1}{4} s(s-1) t(t+1)$ \\
8 & $\frac{1}{2}\left(1-s^{2}\right) t(t+1)$ \\
9 & $\frac{1}{4} s(s+1) t(t+1)$ \\
\hline
\end{tabular}

Table 1: 


\begin{tabular}{|c|c|c|c|c|c|c|}
\hline & \multicolumn{6}{|c|}{ Geometric Mean $\stackrel{\times}{\div}$ Standard Deviation of Relative Error } \\
\hline$\phi_{i}$ & Telles $(20)$ & $\log -\mathrm{L}_{1}(10)$ & Polar $(10)$ & Iteration $0(20)$ & Iteration $1(20)$ & Iteration $2(20)$ \\
\hline \multirow[t]{2}{*}{1} & $3.1 \times 10^{-1}$ & $2.1 \times 10^{-3}$ & $1.9 \times 10^{-1}$ & $4.9 \times 10^{-1}$ & $2.1 \times 10^{-3}$ & $2.0 \times 10^{-4}$ \\
\hline & $\stackrel{\times}{\div} 15.2$ & $\stackrel{\times}{\div} 8.2$ & $\stackrel{\times}{\div} 40.9$ & $\stackrel{\times}{\div} 5.4$ & $\stackrel{\times}{\div} 5.2$ & $\stackrel{\times}{\div} 8.7$ \\
\hline \multirow[t]{2}{*}{2,4} & $5.3 \times 10^{-1}$ & $2.6 \times 10^{-3}$ & $4.0 \times 10^{-1}$ & $6.4 \times 10^{-1}$ & $2.5 \times 10^{-3}$ & $1.2 \times 10^{-4}$ \\
\hline & $\stackrel{\times}{\div} 7.8$ & $\stackrel{\times}{\div} 11.1$ & $\stackrel{\times}{\div} 13.6$ & $\stackrel{\times}{\div} 3.9$ & $\stackrel{\times}{\div} 5.5$ & $\stackrel{\times}{\div} 5.6$ \\
\hline \multirow[t]{2}{*}{3,7} & $2.9 \times 10^{-1}$ & $1.8 \times 10^{-3}$ & $1.8 \times 10^{-1}$ & $4.4 \times 10^{-1}$ & $1.9 \times 10^{-3}$ & $1.8 \times 10^{-4}$ \\
\hline & $\stackrel{\times}{\div} 16.9$ & $\stackrel{\times}{\div} 11.8$ & $\stackrel{\times}{\div} 43.0$ & $\stackrel{\times}{\div} 6.1$ & $\stackrel{\times}{\div} 6.0$ & $\stackrel{\times}{\div} 7.2$ \\
\hline \multirow[t]{2}{*}{5} & $1.0 \times 10^{+00}$ & $5.9 \times 10^{-3}$ & $9.6 \times 10^{-1}$ & $9.9 \times 10^{-1}$ & $4.0 \times 10^{-3}$ & $1.1 \times 10^{-4}$ \\
\hline & $\stackrel{\times}{\div} 1.5$ & $\stackrel{\times}{\div} 1.3$ & $\stackrel{\times}{\div} 1.0$ & $\stackrel{\times}{\div} 1.0$ & $\stackrel{\times}{\div} 3.2$ & $\stackrel{\times}{\div} 5.0$ \\
\hline \multirow[t]{2}{*}{6,8} & $5.3 \times 10^{-1}$ & $2.6 \times 10^{-3}$ & $4.0 \times 10^{-1}$ & $6.4 \times 10^{-1}$ & $2.5 \times 10^{-3}$ & $1.3 \times 10^{-4}$ \\
\hline & $\stackrel{\times}{\div} 7.8$ & $\stackrel{\times}{\div} 11.1$ & $\stackrel{\times}{\div} 13.6$ & $\stackrel{\times}{\div} 3.9$ & $\stackrel{\times}{\div} 5.5$ & $\stackrel{\times}{\div} 5.1$ \\
\hline \multirow[t]{2}{*}{9} & $2.7 \times 10^{-1}$ & $1.6 \times 10^{-3}$ & $1.6 \times 10^{-1}$ & $4.0 \times 10^{-1}$ & $1.8 \times 10^{-3}$ & $1.7 \times 10^{-4}$ \\
\hline & $\stackrel{\times}{\div} 18.7$ & $\stackrel{\times}{\div} 16.0$ & $\stackrel{\times}{\div} 52.4$ & $\stackrel{\times}{\div} 6.8$ & $\stackrel{\times}{\div} 6.9$ & $\stackrel{\times}{\div} 5.3$ \\
\hline
\end{tabular}

Table 2: 


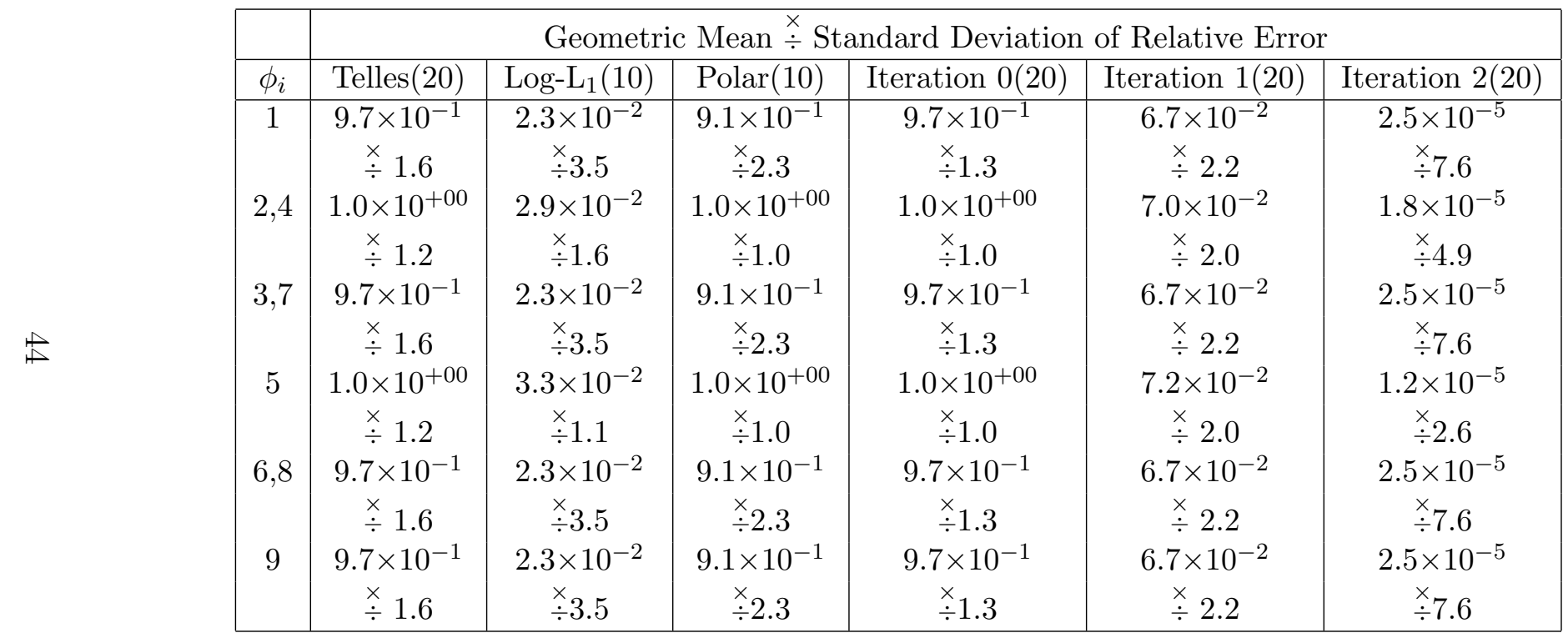

Table 3: 


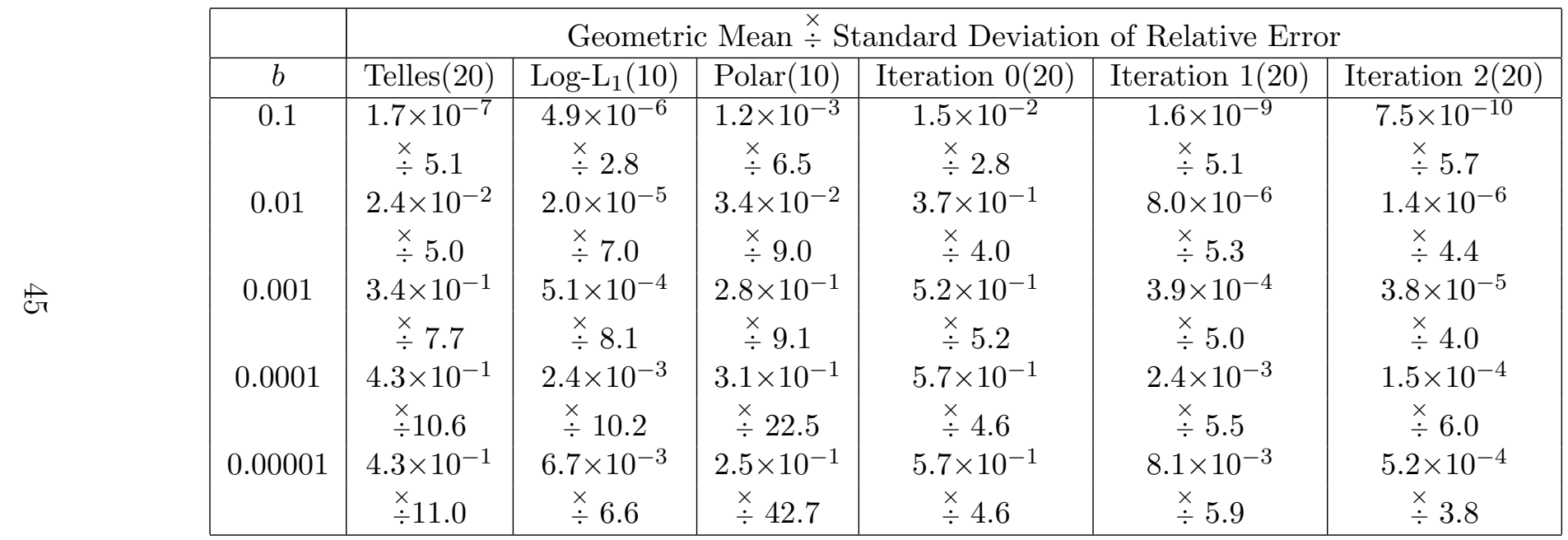

Table 4: 


\begin{tabular}{|c|c|c|c|c|c|c|c|}
\hline \multirow{2}{*}{\multicolumn{2}{|c|}{$b$}} & \multicolumn{6}{|c|}{ Geometric Mean $\stackrel{\times}{\div}$ Standard Deviation of Relative Error } \\
\hline & & Telles(20) & $\log -\mathrm{L}_{1}(10)$ & Polar(10) & Iteration $0(20)$ & Iteration $1(20)$ & Iteration $2(20)$ \\
\hline \multirow{10}{*}{ S } & 0.1 & $1.4 \times 10^{-6}$ & $1.2 \times 10^{-5}$ & $9.5 \times 10^{-3}$ & $6.9 \times 10^{-2}$ & $2.3 \times 10^{-7}$ & $2.8 \times 10^{-10}$ \\
\hline & & $\stackrel{\times}{\div} 4.4$ & $\stackrel{\times}{\div} 2.8$ & $\stackrel{\times}{\div} 2.0$ & $\stackrel{\times}{\div} 2.6$ & $\stackrel{\times}{\div} 4.4$ & $\stackrel{\times}{\div} 4.1$ \\
\hline & 0.01 & $1.6 \times 10^{-1}$ & $2.0 \times 10^{-3}$ & $4.8 \times 10^{-2}$ & $8.6 \times 10^{-1}$ & $6.5 \times 10^{-4}$ & $3.2 \times 10^{-7}$ \\
\hline & & $\stackrel{\times}{\div} 2.7$ & $\stackrel{\times}{\div} 1.5$ & $\stackrel{\times}{\div} 3.6$ & $\stackrel{\times}{\div} 1.3$ & $\stackrel{\times}{\div} 2.5$ & $\stackrel{\times}{\div} 5.4$ \\
\hline & 0.001 & $9.5 \times 10^{-1}$ & $6.7 \times 10^{-3}$ & $8.4 \times 10^{-1}$ & $9.8 \times 10^{-1}$ & $1.4 \times 10^{-2}$ & $3.7 \times 10^{-6}$ \\
\hline & & $\stackrel{\times}{\div} 1.8$ & $\stackrel{\times}{\div} 1.8$ & $\stackrel{\times}{\div} 1.6$ & $\stackrel{\times}{\div} 1.2$ & $\stackrel{\times}{\div} 2.8$ & $\stackrel{\times}{\div} 9.2$ \\
\hline & 0.0001 & $1.0 \times 10^{0}$ & $2.6 \times 10^{-2}$ & $9.6 \times 10^{-1}$ & $9.9 \times 10^{-1}$ & $6.9 \times 10^{-2}$ & $2.0 \times 10^{-5}$ \\
\hline & & $\stackrel{\times}{\div} 1.4$ & $\stackrel{\times}{\div} 2.5$ & $\stackrel{\times}{\div} 1.8$ & $\stackrel{\times}{\div} 1.2$ & $\stackrel{\times}{\div} 2.1$ & $\stackrel{\times}{\div} 5.8$ \\
\hline & 0.00001 & $9.8 \times 10^{-1}$ & $6.8 \times 10^{-3}$ & $9.5 \times 10^{-1}$ & $9.9 \times 10^{-1}$ & $1.5 \times 10^{-1}$ & $5.4 \times 10^{-5}$ \\
\hline & & $\stackrel{\times}{\div} 1.4$ & $\stackrel{\times}{\div} 4.2$ & $\stackrel{\times}{\div} 2.0$ & $\stackrel{\times}{\div} 1.2$ & $\stackrel{\times}{\div} 2.6$ & $\stackrel{\times}{\div} 5.1$ \\
\hline
\end{tabular}

Table 5: 


\begin{tabular}{|c|c|c|c|c|c|c|}
\hline Integral & $b$ & Telles(10) & $\log _{-} \mathrm{L}_{1}(5)$ & $\mathrm{L}^{-1 / 5}(5)$ & Iteration $1(10)$ & Iteration $2(10)$ \\
\hline \multirow{9}{*}{$I_{3 \mathrm{~A}, 1, x}$} & 10 & $1.7431 \times 10^{-13}$ & $4.1788 \times 10^{-5}$ & $4.7961 \times 10^{-5}$ & $8.1198 \times 10^{-14}$ & $5.0528 \times 10^{-14}$ \\
\hline & 3 & $8.7514 \times 10^{-15}$ & $8.8536 \times 10^{-5}$ & $2.6482 \times 10^{-4}$ & $9.8333 \times 10^{-14}$ & $1.2915 \times 10^{-11}$ \\
\hline & 1 & $1.0735 \times 10^{-10}$ & $2.8448 \times 10^{-5}$ & $1.9485 \times 10^{-3}$ & $3.2292 \times 10^{-10}$ & $2.5557 \times 10^{-7}$ \\
\hline & 0.3 & $1.4505 \times 10^{-6}$ & $1.7843 \times 10^{-4}$ & $8.2978 \times 10^{-3}$ & $3.0703 \times 10^{-7}$ & $6.2979 \times 10^{-5}$ \\
\hline & 0.1 & $6.0415 \times 10^{-4}$ & $8.7729 \times 10^{-4}$ & $1.8437 \times 10^{-2}$ & $7.1078 \times 10^{-5}$ & $7.7113 \times 10^{-4}$ \\
\hline & 0.03 & $2.3479 \times 10^{-2}$ & $1.0892 \times 10^{-3}$ & $8.2145 \times 10^{-3}$ & $2.5949 \times 10^{-3}$ & $1.1681 \times 10^{-3}$ \\
\hline & 0.01 & $3.7817 \times 10^{-2}$ & $1.9475 \times 10^{-2}$ & $3.2038 \times 10^{-3}$ & $6.3656 \times 10^{-3}$ & $1.1911 \times 10^{-3}$ \\
\hline & 0.003 & $4.7819 \times 10^{-1}$ & $3.1602 \times 10^{-2}$ & $5.5507 \times 10^{-3}$ & $3.4189 \times 10^{-4}$ & $5.9140 \times 10^{-5}$ \\
\hline & 0.001 & $7.1627 \times 10^{-1}$ & $2.2359 \times 10^{-3}$ & $2.5207 \times 10^{-3}$ & $3.7283 \times 10^{-3}$ & $5.5117 \times 10^{-3}$ \\
\hline Integral & $b$ & Telles(20) & $\log _{-} \mathrm{L}_{1}(10)$ & $\mathrm{L}^{-1 / 5}(10)$ & Iteration $1(20)$ & Iteration $2(20)$ \\
\hline \multirow[t]{9}{*}{$I_{3 \mathrm{~A}, 1, x}$} & 10 & $3.6230 \times 10^{-13}$ & $9.5864 \times 10^{-12}$ & $9.5553 \times 10^{-12}$ & $3.6098 \times 10^{-13}$ & $3.6274 \times 10^{-13}$ \\
\hline & 3 & $8.3854 \times 10^{-14}$ & $1.1988 \times 10^{-10}$ & $1.1920 \times 10^{-10}$ & $8.4331 \times 10^{-14}$ & $8.3854 \times 10^{-14}$ \\
\hline & 1 & $1.0453 \times 10^{-14}$ & $2.2481 \times 10^{-9}$ & $1.9030 \times 10^{-8}$ & $1.0201 \times 10^{-14}$ & $9.3191 \times 10^{-15}$ \\
\hline & 0.3 & $6.4266 \times 10^{-14}$ & $2.4481 \times 10^{-8}$ & $8.8894 \times 10^{-8}$ & $3.5878 \times 10^{-14}$ & $3.7138 \times 10^{-10}$ \\
\hline & 0.1 & $7.3894 \times 10^{-8}$ & $1.8620 \times 10^{-6}$ & $2.8312 \times 10^{-6}$ & $1.7784 \times 10^{-9}$ & $1.2061 \times 10^{-8}$ \\
\hline & 0.03 & $1.2939 \times 10^{-4}$ & $1.0820 \times 10^{-5}$ & $1.0695 \times 10^{-5}$ & $5.4582 \times 10^{-7}$ & $2.7759 \times 10^{-7}$ \\
\hline & 0.01 & $1.7441 \times 10^{-4}$ & $8.4758 \times 10^{-5}$ & $6.4723 \times 10^{-6}$ & $1.1295 \times 10^{-5}$ & $4.3083 \times 10^{-6}$ \\
\hline & 0.003 & $3.0799 \times 10^{-2}$ & $4.9795 \times 10^{-5}$ & $1.2466 \times 10^{-4}$ & $3.2861 \times 10^{-5}$ & $1.6573 \times 10^{-5}$ \\
\hline & 0.001 & $1.0791 \times 10^{-1}$ & $1.0583 \times 10^{-3}$ & $2.3023 \times 10^{-4}$ & $1.3832 \times 10^{-3}$ & $2.3762 \times 10^{-5}$ \\
\hline
\end{tabular}

Table 6: 


\begin{tabular}{|c|c|c|c|c|c|c|}
\hline Integral & $b$ & Telles $(10)$ & Log- $_{1}(5)$ & $\mathrm{L}^{-1 / 5}(5)$ & Iteration 1(10) & Iteration 2(10) \\
\hline$I_{3 \mathrm{~B}, 1, x}$ & 10 & $3.3123 \times 10^{-11}$ & $2.4413 \times 10^{-5}$ & $2.9952 \times 10^{-5}$ & $3.3049 \times 10^{-11}$ & $3.2936 \times 10^{-11}$ \\
& 3 & $1.1585 \times 10^{-13}$ & $1.1342 \times 10^{-4}$ & $9.9254 \times 10^{-5}$ & $2.4271 \times 10^{-14}$ & $3.6395 \times 10^{-12}$ \\
& 1 & $1.3607 \times 10^{-9}$ & $1.3558 \times 10^{-3}$ & $2.0562 \times 10^{-3}$ & $1.6096 \times 10^{-9}$ & $4.2631 \times 10^{-7}$ \\
& 0.3 & $4.1983 \times 10^{-6}$ & $3.0214 \times 10^{-3}$ & $1.6505 \times 10^{-3}$ & $2.7201 \times 10^{-6}$ & $2.6417 \times 10^{-5}$ \\
& 0.1 & $5.3330 \times 10^{-4}$ & $5.5639 \times 10^{-3}$ & $1.7007 \times 10^{-3}$ & $2.9927 \times 10^{-4}$ & $3.9747 \times 10^{-4}$ \\
& 0.03 & $1.9774 \times 10^{-2}$ & $2.3587 \times 10^{-2}$ & $1.7770 \times 10^{-2}$ & $6.1684 \times 10^{-3}$ & $1.0998 \times 10^{-3}$ \\
& 0.01 & $3.3880 \times 10^{-1}$ & $5.5849 \times 10^{-2}$ & $5.1134 \times 10^{-4}$ & $2.0069 \times 10^{-2}$ & $5.5327 \times 10^{-4}$ \\
& 0.003 & $7.9890 \times 10^{-1}$ & $5.6386 \times 10^{-2}$ & $6.0018 \times 10^{-2}$ & $3.9846 \times 10^{-2}$ & $1.1033 \times 10^{-2}$ \\
& 0.001 & $6.7698 \times 10^{-1}$ & $2.1793 \times 10^{-1}$ & $1.0631 \times 10^{-1}$ & $5.3260 \times 10^{-3}$ & $2.8335 \times 10^{-2}$ \\
\hline \multirow{5}{*}{ Integral } & $b$ & Telles $(20)$ & Log-L $(10)$ & $\mathrm{L}^{-1 / 5}(10)$ & Iteration $1(20)$ & Iteration $2(20)$ \\
\hline$I_{3 \mathrm{~B}, 1, x}$ & 10 & $3.2552 \times 10^{-11}$ & $4.7012 \times 10^{-11}$ & $4.6963 \times 10^{-11}$ & $3.2551 \times 10^{-11}$ & $3.2555 \times 10^{-11}$ \\
& 3 & $1.5297 \times 10^{-14}$ & $1.4244 \times 10^{-10}$ & $1.8809 \times 10^{-10}$ & $1.5297 \times 10^{-14}$ & $1.5297 \times 10^{-14}$ \\
& 1 & $1.3129 \times 10^{-14}$ & $4.6434 \times 10^{-9}$ & $5.1904 \times 10^{-9}$ & $1.1078 \times 10^{-14}$ & $1.3129 \times 10^{-14}$ \\
& 0.3 & $1.4638 \times 10^{-14}$ & $1.1676 \times 10^{-7}$ & $1.2628 \times 10^{-7}$ & $1.8148 \times 10^{-13}$ & $1.3637 \times 10^{-10}$ \\
& 0.1 & $4.0820 \times 10^{-8}$ & $4.3757 \times 10^{-6}$ & $3.8950 \times 10^{-6}$ & $1.2296 \times 10^{-8}$ & $1.8985 \times 10^{-8}$ \\
& 0.03 & $7.1541 \times 10^{-4}$ & $6.5019 \times 10^{-5}$ & $2.5165 \times 10^{-5}$ & $3.5820 \times 10^{-6}$ & $8.5297 \times 10^{-8}$ \\
& 0.01 & $8.4637 \times 10^{-3}$ & $2.3575 \times 10^{-4}$ & $1.4487 \times 10^{-4}$ & $2.7848 \times 10^{-5}$ & $4.5533 \times 10^{-6}$ \\
& 0.003 & $3.9623 \times 10^{-2}$ & $2.5753 \times 10^{-3}$ & $4.0308 \times 10^{-4}$ & $1.8176 \times 10^{-3}$ & $8.4782 \times 10^{-6}$ \\
& 0.001 & $4.1205 \times 10^{-1}$ & $2.1830 \times 10^{-3}$ & $2.5457 \times 10^{-4}$ & $7.5430 \times 10^{-3}$ & $5.9369 \times 10^{-6}$ \\
\hline
\end{tabular}

Table 7: 


\begin{tabular}{|c|c|c|c|c|c|c|}
\hline Integral & $b$ & Telles $(20)$ & Log- $_{1}(10)$ & $\mathrm{L}^{-1 / 5}(10)$ & Iteration 1(20) & Iteration 2(20) \\
\hline$I_{2 \mathrm{~A}, 1}$ & 10 & $1.9085 \times 10^{-14}$ & $1.3635 \times 10^{-11}$ & $1.3633 \times 10^{-11}$ & $1.9085 \times 10^{-14}$ & $1.8388 \times 10^{-14}$ \\
& 3 & $6.8774 \times 10^{-15}$ & $2.1604 \times 10^{-11}$ & $1.8548 \times 10^{-11}$ & $7.8152 \times 10^{-15}$ & $8.2841 \times 10^{-15}$ \\
& 1 & $1.1603 \times 10^{-14}$ & $4.7961 \times 10^{-11}$ & $9.4826 \times 10^{-11}$ & $1.0210 \times 10^{-14}$ & $8.2379 \times 10^{-15}$ \\
& 0.3 & $1.6959 \times 10^{-14}$ & $2.1548 \times 10^{-8}$ & $4.5478 \times 10^{-7}$ & $2.0115 \times 10^{-14}$ & $1.3571 \times 10^{-11}$ \\
& 0.1 & $6.0008 \times 10^{-10}$ & $7.8896 \times 10^{-7}$ & $4.3975 \times 10^{-6}$ & $1.1522 \times 10^{-11}$ & $2.6816 \times 10^{-8}$ \\
& 0.03 & $7.0469 \times 10^{-7}$ & $9.7374 \times 10^{-6}$ & $6.3698 \times 10^{-4}$ & $2.6839 \times 10^{-9}$ & $8.3058 \times 10^{-6}$ \\
& 0.01 & $2.0531 \times 10^{-5}$ & $2.4049 \times 10^{-5}$ & $8.6511 \times 10^{-3}$ & $2.6908 \times 10^{-8}$ & $1.0875 \times 10^{-4}$ \\
& 0.003 & $6.7337 \times 10^{-5}$ & $9.7945 \times 10^{-5}$ & $5.5216 \times 10^{-2}$ & $2.5833 \times 10^{-7}$ & $4.6281 \times 10^{-4}$ \\
& 0.001 & $1.4848 \times 10^{-4}$ & $7.6522 \times 10^{-4}$ & $1.7480 \times 10^{-1}$ & $3.2865 \times 10^{-6}$ & $9.0639 \times 10^{-4}$ \\
\hline
\end{tabular}

Table 8: 


\begin{tabular}{|c|c|c|c|c|c|c|c|c|c|c|}
\hline \multicolumn{11}{|c|}{ Time in Seconds to Evaluate Integrals 10000 Times } \\
\hline & \multicolumn{5}{|c|}{ Potential Integral $I_{2 \mathrm{~A}, 1}$} & \multicolumn{5}{|c|}{ Flux Integral $I_{3 \mathrm{~B}, 1, x}$} \\
\hline $\mathrm{b}$ & Telles & $\mathrm{Log}-\mathrm{L}_{1}$ & $\mathrm{~L}_{1}^{-1 / 5}$ & Iteration 1 & Iteration 2 & Telles & $\log -\mathrm{L}_{1}$ & $\mathrm{~L}_{1}^{-1 / 5}$ & Iteration 1 & Iteration 2 \\
\hline 10 & 0.160 & 0.623 & 0.731 & 0.104 & 0.119 & 0.190 & 0.901 & 1.043 & 0.121 & 0.118 \\
\hline 3 & 0.164 & 0.624 & 0.830 & 0.125 & 0.143 & 0.231 & 1.017 & 1.176 & 0.144 & 0.188 \\
\hline 1 & 0.262 & 0.802 & 0.935 & 0.170 & 0.311 & 0.421 & 1.489 & 1.860 & 0.303 & 0.311 \\
\hline 0.3 & 0.679 & 1.112 & 1.304 & 0.450 & 0.693 & 0.806 & 1.123 & 1.436 & 0.486 & 0.507 \\
\hline 0.1 & 1.210 & 0.990 & 1.268 & 0.621 & 1.156 & 1.741 & 1.192 & 1.279 & 0.812 & 0.919 \\
\hline 0.03 & 3.027 & 1.128 & 1.607 & 1.029 & 1.743 & 4.517 & 1.312 & 1.598 & 1.268 & 1.235 \\
\hline 0.01 & 7.061 & 1.638 & 2.536 & 1.548 & 2.344 & 9.959 & 1.674 & 1.504 & 1.773 & 1.390 \\
\hline 0.003 & 16.411 & 1.755 & 3.031 & 1.544 & 2.929 & 21.618 & 1.491 & 1.584 & 2.531 & 1.919 \\
\hline 0.001 & 27.809 & 1.873 & 3.442 & 3.116 & 3.492 & 41.974 & 1.793 & 1.706 & 3.560 & 2.190 \\
\hline
\end{tabular}

Table 9: 